\title{
A FAST INJECTION KICKER MAGNET FOR THE TEVATRON
}

\begin{abstract}
A new proton injection kicker system is required for the Tevatron in the Run II era. The new system was designed to supply $1.25 \mathrm{kG}-\mathrm{m}$ into a magnetic aperture of $48 \mathrm{~mm}$ vertical x $71 \mathrm{~mm}$ horizontal x $5 \mathrm{~m}$ long with a $396 \mathrm{~ns}$ bunch spacing. The system is configured to be upgraded to a bunch spacing of $132 \mathrm{~ns}$ with additional pulsed power supplies. The design of the magnet incorporated some novel features in order to meet these requirements. These include adjustable bus spacing to set the inductance, balanced positive and negative high voltage buses and an integrated capacitive pickup. This system has been installed in the Tevatron since July 2000.
\end{abstract}

C. Jensen, B. Hanna, R. Reilly, Fermilab*, Batavia IL USA 60510

\section{INTRODUCTION}

The Tevatron for Run II requires 36 proton x 36 pbar bunch stores with a $376 \mathrm{~ns}$ rise time and a 2.6 us fall time. The pbar injection kicker was successfully upgraded in 1995, but the proton injection kicker was not. To allow for even higher luminosity, it was decided to build the proton injection kicker with the capability of $132 \mathrm{~ns}$ bunch spacing (112 ns rise time). This was done by installing five magnets in the beam line; fewer could have been used to meet to initial rise time requirements. These shorter magnets are now powered as two systems, with two in series and three in series. When the shorter bunch spacing in needed, additional power supplies will be added and each magnet will be individually powered. The power supplies and system are discussed in a companion paper.

\section{MAGNET DESIGN}

The magnet design posed several mechanical and electrical challenges. There is not much room in the Tevatron lattice and therefore the magnets had to be relatively small. There are obstructions $10 "$ above and below the Tevatron beam line center. Design impedances of $16.7 \mathrm{Ohms}$ and 12.5 Ohms were simulated using SPICE as were balanced and unbalanced systems. The impedance chosen for the best system performance was 12.5 Ohms with balanced positive and negative pulsers each driving $20 \mathrm{kV}$ into matched loads.

First, to optimize the performance, a new ceramic vacuum chamber was designed. The minimum clear aperture for the Tevatron in colliding mode was determined by the machine physicists. Ten new rounded rectangular ceramic tubes were then manufactured and the best six selected for use. Some elements on the final magnet design were determined by the physical dimensions of the ceramic tubes as received, but design work continued.

The second design issue was the capacitors used to accomplish the impedance and segmentation. The value required, $80 \mathrm{pF}$ per cell, bus to bus, could have been

\footnotetext{
* Fermilab is operated by University Research Association Inc. under
} Contract No. DE-AC02-76CH03000 accomplished in two ways. Two capacitors of $40 \mathrm{pF}$ and at least $40 \mathrm{kV}$ could be used in parallel or four capacitors of $80 \mathrm{pF}$ and at least $20 \mathrm{kV}$ could be used in a seriesparallel configuration. Either option would require some capacitor development, in either a higher voltage or lower capacitance. The second option was preferred because there seemed less risk in a ceramic formulation that reduced capacitance than one that increased voltage gradient.

Because of the variability in the nominal capacitance value from batch to batch, the inductance per magnet also had to have some adjustment range. This was accomplished by adjusting the gap between the bus bars to tight tolerances, \pm 0.01 inches. This feature also allowed for the compensation of stray capacitances which would otherwise change the impedance of the magnet. The adjustment range was very useful during the tuning of the magnet impedance. Finally, a minimal equivalent series inductance for the capacitors is required to give low dispersion. This meant the ferrite needed to have a copper foil along the sides to limit this inductance and have cross coupling between adjacent ferrites to further reduce it [1].

\subsection{Magnet Capacitors}

The absolute tolerance on high voltage ceramic capacitors is an industry standard of $\pm 10 \%$. For the pbar injection kicker [2], attempts were made to build a long lifetime parallel plate capacitor. When those attempts failed, several batches of commercially available capacitors were purchased from a manufacturer. These were then measured and sorted in sets of 4 to make the required total capacitance. It was noted at that time that while from batch to batch the capacitance could vary significantly, within a single batch the tolerances were very tight $( \pm 1 \%)$. For this project, the sorting method could not be used effectively for two reasons. First, the capacitance per section is so small that it was less than the standard values. Second, the capacitors in this magnet are used in series between the high voltage buses; there is no common connection. The lack of a common connection is intentional. This is a balanced system and the high voltage buses are strongly coupled. Therefore, when one of the thyratrons pre-fires it drives a common mode and differential mode voltage on the high voltage buses. This results in $\sim 75 \%$ of the open circuit voltage occurring on one bus and $\sim 25 \%$ on the other. The result is the same voltage in either nominal or fault conditions when there is not a capacitor connection to common. With the connection however, a $50 \%$ over voltage would occur in the fault case. This also means the capacitance of each side has to be closely matched to avoid exciting the common mode of the magnet and introducing reflections and coupling between the modes. 
We purchased 100 capacitors with a nominal values of $80 \mathrm{pF}$ and $30 \mathrm{kV}$ from three manufacturers [3],[4],[5] to determine if they would meet the lifetime requirements. Each manufacturer was within the allowed $\pm 10 \%$ nominal value. Tolerances over each build were less than $\pm 0.75 \%$. These capacitors were placed in a prototype magnet and pulsed at $25 \mathrm{kV}$ for $10^{6}$ pulses, longer than the lifetime of the system. All succeeded and we then purchased 1100 capacitors from the vendor with the most timely delivery. These final capacitors were then measured at $1 \mathrm{~V}$ and $1 \mathrm{kHz}$ and sorted into only 3 bins $(79 \mathrm{pF}, 80 \mathrm{pF}$ and $81 \mathrm{pF})$. The average value was used to set the nominal spacing for the bus bars. The capacitors were installed in the magnet in two parallel sets, using capacitors from the same bin in series.

A special end value of capacitance is also used. The value was empirically determined to be about twice the standard cell capacitance. While the exact value of the end capacitor for optimum performance is not known, measurements and simulations of the magnet clearly showed an improvement in the 1\%-99\% field rise time when the end capacitor is $150-200 \%$ of the main value.

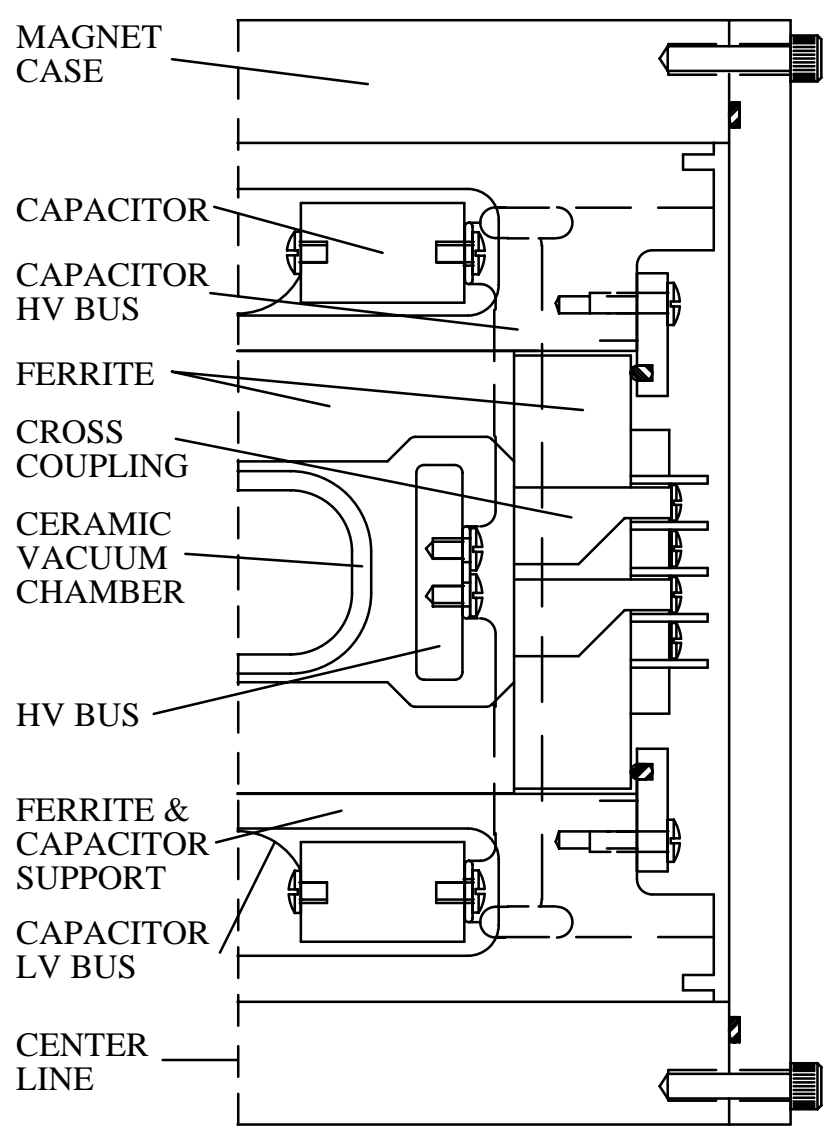

Figure 1. Cross Section of Half of Magnet

\subsection{Magnet Inductance Design}

The setting tolerance on the inductance is $\pm 1 \%$, however a tuning range of approximately $5 \%$ is also required because of the range in average capacitance. As a result of our prototype testing, we knew the approximate values of the stray capacitance and therefore the required inductance of our design.

Given this inductance, a cross section of the magnet was modelled using Opera-2D [6]. An AC simulation at $1 \mathrm{MHz}$ was done of half the magnet and the stored energy was calculated both in the gap and in the rest of the magnet. The same model was then done with the ferrite replaced with air. The energy stored in the magnet, except the region of the air gap, was then calculated for this air filled magnet. The total inductance per cell was then calculated by first multiplying the stored energy of the gap by the cell length (length of ferrite plus length of gap between ferrites). Next, the stored energy of the rest of the magnet when modelled as ferrite times the length of the ferrite was calculated. Finally, the stored energy of the rest of the magnet when modelled as air times the length of the gap between ferrites was calculated and the result of all three calculations was added together and divided by the square of the current. This method resulted in a simulation that included most 3-D effects but was easier to perform. These numbers were compared with measurements and found to be accurate to $\pm 1 \%$, which is about the same accuracy as the simulation.

The simulations also showed that our initial ferrite pole piece design required modification. Since essentially all the flux in the gap (the length of a cell) returns through the ferrite, the flux density in the ferrite is higher than the simulation by the reciprocal of the packing factor, which is 0.73 for this design.

\subsection{Other Design Issues}

One of the hardest problems related to fast kicker systems is measuring the performance. While the final measurement is done with the beam, it is imprudent to rely on this as the system has then been installed and is difficult to change. One of the early modifications we made to the magnet was suggested by John Dinkel (retired Fermilab). We put in a capacitive pickup directly around the input high voltage lead. This pickup is quite fast and accurate. We used this pickup to measure the magnet input and output voltage and from those calculate the integrated field. Without these pickups, the compact nature of the magnet made any high voltage measurements impossible.

The final magnet cross section in shown in Figure1. This view shows only half of the magnet. The full magnet is symmetrical about the center line.

\section{MEASUREMENTS}

After each magnet was assembled, it went through a series of measurements. First, the capacitance and inductance of the magnet were measured in a series of steps. These measurements were then fit to a model of the magnet and the inductance and capacitance per section of magnet were calculated. The capacitance was measured with air dielectric and a Fluorinert FC-40 as a dielectric $\left(\varepsilon_{\mathrm{r}}=1.9\right)$. The distance between the high voltage bus bar 


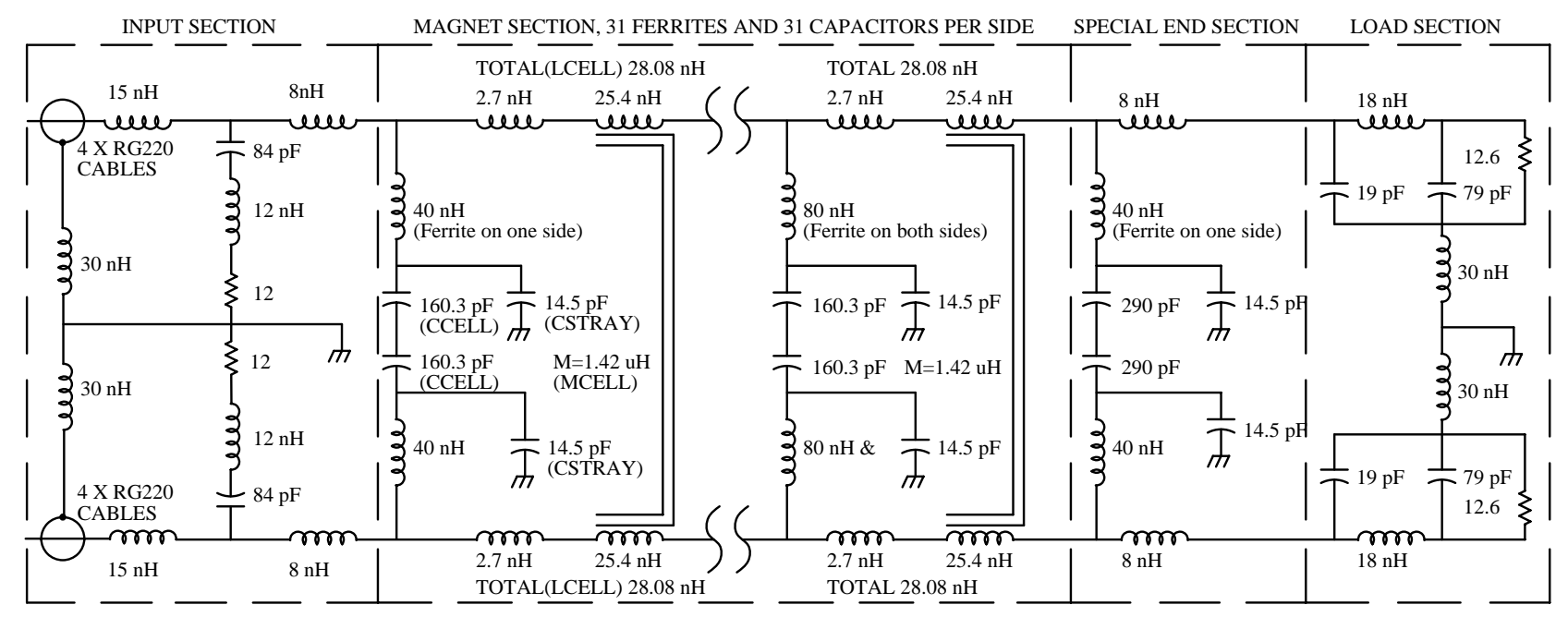

Figure 2, Equivalent Circuit of a Single Magnet, All parameters are based on experimental measurements

Table I. Measured Values of All Magnets

\begin{tabular}{|l|l|l|l|l|}
\hline Magnet & $\begin{array}{l}\text { C/section } \\
\text { (CCELL) }\end{array}$ & $\begin{array}{l}\text { C/section } \\
\text { (CSTRAY) }\end{array}$ & $\begin{array}{l}\text { L/section } \\
\text { (LCELL) }\end{array}$ & Spacing \\
\hline 1 & $160.3 \mathrm{pF}$ & $14.5 \mathrm{pF}$ & $28.1 \mathrm{nH}$ & $2.905 ”$ \\
\hline 2 & $159.8 \mathrm{pF}$ & $14.3 \mathrm{pF}$ & $27.9 \mathrm{nH}$ & $2.909 ”$ \\
\hline 3 & $160.7 \mathrm{pF}$ & $13.8 \mathrm{pF}$ & $28.1 \mathrm{nH}$ & $2.908^{\prime \prime}$ \\
\hline 4 & $160.8 \mathrm{pF}$ & $13.9 \mathrm{pF}$ & $28.1 \mathrm{nH}$ & $2.931 "$ \\
\hline 5 & $162.0 \mathrm{pF}$ & $12.0 \mathrm{pF}$ & $27.9 \mathrm{nH}$ & $2.904 ”$ \\
\hline
\end{tabular}

was also measured. Fitting the measured data to a model of both the air and Fluorinert filled magnet, we then readjusted the spacing on the bus bar to get the required impedance $( \pm 1 / 2 \%)$. It was important to do these measurements at the same temperature as the beam line enclosure as the capacitors have a significant temperature coefficient $(\sim-0.4 \% / \mathrm{C})$. We adjusted the spacing to achieve an impedance of $12.45 \mathrm{Ohms}$ as this gives a slight initial overshoot and results in a several ns reduction in the field rise time. Table I shows the measured parameters for all 5 installed magnets and Figure 2 shows the magnet equivalent circuit.

Our last measurements were the input and output voltage measurements. We used the capacitive dividers to measure the input and output voltage with a LeCroy LC584 oscilloscope. Then, using a spreadsheet, we subtracting the input from the output voltage and numerically integrated over the pulse. This was done for a single magnet (for the $113 \mathrm{~ns}$ rise time requirement) and for the two and three magnet systems (for the $372 \mathrm{~ns}$ rise time). The response is shown in Figure 3. The two magnet and three magnet system have load resistors of 12.55 and $12.62 \mathrm{Ohms}$, respectively, and the difference in response can be seen.

We have met our all the specifications and the system has been running in the Tevatron for since July 2000. We anticipate installing the additional pulse supplies and reconfiguring the magnets before the start of Run IIb.

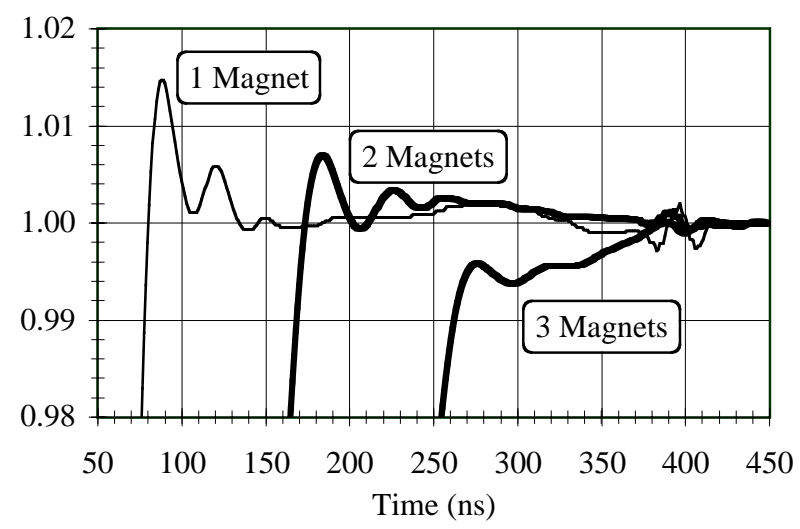

Figure 3, Normalized Integrated Field vs. Time, Expanded Vertical Scale, Zero Time is set to 1\% of Flattop

\section{REFERENCE}

[1] G. Nassibian, "Traveling Wave Kicker Magnets with Sharp Rise and Less Overshoot", IEEE Trans. Nucl. Sci., Vol 26,pp.4018-4020,1979

[2] J. Dinkel, et. al., "Development of a High Quality Kicker Magnet System", PAC 1993, Washington May 1993, pp1357-1359

[3] Murate Electronics Products, Smyrna GA 30080

[4] High Energy Capacitor, Parkesburg PA 19365

[5] Ceramite, Grafton WI, 53024

[6] Opera-2D, Vers. 1.5, Vector Fields, Aurora IL 60505 